\section{Analysis of Effectiveness of Lyapunov Control for Non-generic Quantum States}

\author{
Xiaoting Wang* and S. G. Schirmer*† \\ * Dept of Applied Maths \& Theoretical Physics \\ Univ. of Cambridge, Wilberforce Rd, Cambridge, CB3 0WA, UK \\ Email: x.wang@damtp.cam.ac.uk,sgs29@cam.ac.uk
}

\begin{abstract}
A Lyapunov-based control design for natural trajectorytracking problems is analyzed for quantum states where the analysis in the generic case is not applicable. Using dynamical systems tools we show almost global asymptotic stability for stationary target states subject to certain conditions on the Hamiltonians, and discuss effectiveness of the design when these conditions are not satisfied. For pseudo-pure target states the effectiveness of the design is studied further for both stationary and non-stationary states using alternative tools.
\end{abstract}

\section{INTRODUCTION}

Recent technological advances have prompted significant interest in developing the foundations of quantum control theory. One major concern is how to design the dynamics to steer the system state to a stationary target state, or track the natural trajectory of nonstationary states. Numerous results have been obtained on the theory of Lyapunov-based control [1]-[11] and the references in [11]. Most papers have used the Hilbert-Schmidt (HS) distance as the most natural Lyapunov function and in this setting [4]-[6] showed that the target state is asymptotically stable under a sufficient condition equivalent to controllability of the linearized system for pure states represented by wavefunctions, although an additional control had to be added to fix the relative phase of the state. An alternative design based on an implicit Lyapunov function was proposed in [7] to render (pure) target states asymptotically stable when the linearized system is not controllable. The more general case of systems whose states must be represented by density operators was recently considered in [9], [10], but our analysis for generic quantum states [12], for instance, showed the dynamical landscape and convergence behavior to be more complicated than described in [10]. The aim of this TechNote is to extend this analysis to non-generic states, which, although a set of measure zero, deserve special consideration as they include important cases such as pseudo-pure states.

Specifically we show that, although the LaSalle invariant set for non-generic states is much larger than in the generic case, for ideal systems the target state not only remains isolated and thus locally asymptotically stable, but a dynamical systems analysis for stationary target states shows that all other critical manifolds are unstable and attract at most a measure-zero subset of the state space. At least for the stationary target states, these arguments rigorously show that we indeed have almost global convergence as claimed in [10], although the set of states not attracted to the target state is significantly larger than claimed in [10]. The conditions for effectiveness of the method for stationary target states are similar to those for generic states, and we again find that center manifolds emerge around the target state when these conditions are not satisfied, but in contrast to the generic case, the emergence of a center manifold now is conditional on the target state, i.e., the method may still be effective for some target states even if the ideal Hamiltonian criteria are not satisfied. For the special class of pseudo-pure states, we can further show explicitly that for systems with ideal Hamiltonians, the method is effective not only for stationary target states but also for almost all non-stationary ones. This is similar to the situation for the generic case but in this special case we can characterize the non-regular target states explicitly, which shows that they include some states of special interest such as socalled CAT states and maximally entangled states.

\section{LYAPUNOV CONTROL AND LASALLE INVARIANT SET}

We study the bilinear Hamiltonian control problem for a quantum system on an $n$-dimensional Hilbert space $\mathcal{H}$. The state of such a system is generally represented by a trace-one positive operator $\rho$ on $\mathcal{H}$, referred to as density operator, whose evolution is governed by the quantum Liouville equation

$$
\dot{\rho}(t)=-i\left[H_{0}+f(t) H_{1}, \rho(t)\right],
$$

where $f(t)$ is an admissible real-valued control, and $H_{0}$ and $H_{1}$ are time-independent Hamiltonians corresponding to free evolution and control interaction terms, respectively.

Definition II.1. A density operator $\rho$ represents a pure state if it is a rank-one projector, and a mixed state otherwise; $\rho$ is generic if it has $n$ distinct eigenvalues, and pseudo-pure if its spectrum has only two distinct eigenvalues occurring with multiplicities 1 and $n-1$.

The control problem is to design a control $f(t)$ such that the system state $\rho(t)$ converges to the target state $\rho_{d}$ as $t \rightarrow \infty$. For Hamiltonian evolution a necessary condition for $\rho_{d}$ to be reachable from an initial state $\rho(0)$ is that both $\rho(0)$ and $\rho_{d}$ have the same spectrum, and we shall assume this condition to be satisfied here. The set of density operators isospectral with $\rho_{d}$ forms a compact manifold $\mathcal{M}$, whose dimension depends on the spectrum of $\rho_{d} . \rho_{d}$ is a stationary state of the system if and only if $\left[H_{0}, \rho_{d}\right]=0$. If $\rho_{d}$ is not a stationary state then the control problem becomes a trajectory-tracking problem, and to be able to apply LaSalle's invariance principle to this case, we formulate the control dynamics on an extended state space $\mathcal{M} \times \mathcal{M}$ :

$$
\begin{aligned}
\dot{\rho}(t) & =-i\left[H_{0}+f\left(\rho, \rho_{d}\right) H_{1}, \rho(t)\right], \\
\dot{\rho}_{d}(t) & =-i\left[H_{0}, \rho_{d}(t)\right], \\
f\left(\rho, \rho_{d}\right) & =\operatorname{Tr}\left(\left[-i H_{1}, \rho(t)\right] \rho_{d}(t)\right),
\end{aligned}
$$

where the control function $f$ is chosen such that

$$
V\left(\rho, \rho_{d}\right)=\frac{1}{2} \operatorname{Tr}\left[\left(\rho-\rho_{d}\right)^{2}\right]=\operatorname{Tr}\left(\rho_{d}^{2}\right)-\operatorname{Tr}\left(\rho \rho_{d}\right)
$$

is non-increasing along any flow, i.e.,

$$
\frac{d}{d t} V\left(\rho, \rho_{d}\right)=-f(t) \operatorname{Tr}\left(\rho_{d}\left[-i H_{1}, \rho\right]\right)=-f^{2}(t) \leq 0 .
$$

Thus, the dynamical system $\left(\rho(t), \rho_{d}(t)\right) \in \mathcal{M} \times \mathcal{M}$ is autonomous, all solutions are bounded, and $V\left(\rho_{1}, \rho_{2}\right)$ is a Lyapunov function on $\mathcal{M} \times \mathcal{M}$. From LaSalle's invariance principle [13] we have:

Theorem II.1. Any evolution $\left(\rho(t), \rho_{d}(t)\right)$ for the dynamical system (2) will converge to the LaSalle invariant set, $E=\left\{\left(\rho_{1}, \rho_{2}\right) \in\right.$ $\left.\overline{\mathcal{M}} \times \mathcal{M} \mid \dot{V}\left(\rho(t), \rho_{d}(t)\right)=0,\left(\rho(0), \rho_{d}(0)\right)=\left(\rho_{1}, \rho_{2}\right)\right\}$.

The effectiveness of the control in steering the system towards the target state depends on the asymptotic stability of $\rho_{d}$. Local asymptotic stability is a prerequisite for almost global convergence, and a necessary and sufficient condition for the former is that the target state be isolated in $E$. Hence, we first characterize the invariant set $E$, which depends on both the Hamiltonians $H_{0}, H_{1}$ and $\rho_{d}$.

Definition II.2. Choose a basis such that $H_{0}=\operatorname{diag}\left(a_{1}, \ldots, a_{n}\right)$, which is always possible as $H_{0}$ is Hermitian. Let $H_{1}=\left(b_{k \ell}\right)$ and $\omega_{k \ell}=a_{k}-a_{\ell}$ be the transition frequencies. System (2) is ideal if

(i) $H_{0}$ is strongly regular, i.e., $\omega_{k \ell} \neq \omega_{p q}$ unless $(k, \ell)=(p, q)$.

(ii) $H_{1}$ is fully connected, i.e., $b_{k \ell} \neq 0$ except (possibly) for $k=\ell$.

As shown in [12], we have

Theorem II.2. The invariant set of (2) for an ideal system satisfies $E=\left\{\left(\rho_{1}, \rho_{2}\right) \in \mathcal{M} \times \mathcal{M}:\left[\rho_{1}, \rho_{2}\right]=\operatorname{diag}\left(c_{1}, \ldots, c_{n}\right)\right\}$. 


\section{STATIONARY TARGET STATE}

For a generic stationary state $\rho_{d}$, it has been shown that the LaSalle invariant set $E$ is comprised of $n$ ! distinct stationary states, which coincide with the $n$ ! critical points of $V(\rho)=V\left(\rho, \rho_{d}\right)$ as a Morse function [12]. If $\rho_{d}$ is not generic then $E$ is much larger and the topology becomes quite complicated. If $\rho_{d}$ is stationary then (2) can be reduced to an autonomous system on $\mathcal{M}$, and the LaSalle invariant set to $E=\left\{\rho:\left[\rho, \rho_{d}\right]=0\right\}$. We can also choose a basis such that $H_{0}$ and $\rho_{d}$ are simultaneously diagonal, $H_{0}=\operatorname{diag}\left(a_{1}, \ldots, a_{n}\right)$,

$$
\rho_{d}=\operatorname{diag}\left(w_{1}, \ldots, w_{1}, \ldots, w_{k}, \ldots, w_{k}\right),
$$

where $\left\{w_{\ell}\right\}_{\ell=1}^{k}$ are the distinct eigenvalues of $\rho_{d}$ with multiplicities $n_{\ell}$ and $\sum_{\ell=1}^{k} n_{\ell}=n$. The state space in this case is the flag manifold

$$
\mathcal{M} \simeq \mathbb{U}(n) /\left[\mathbb{U}\left(n_{1}\right) \times \ldots \times \mathbb{U}\left(n_{k}\right)\right]
$$

of dimension $n_{\mathcal{M}}=n^{2}-\sum_{\ell=1}^{k} n_{\ell}^{2}$.

Definition III.1. $x_{0}$ is a critical point of $f(x)$ if $\left.\nabla f(x)\right|_{x=x_{0}}=0$; it is hyperbolic, if the Hessian at the critical point is nonsingular. $f$ is a Morse function if all of the its critical points are hyperbolic.

For a given $\rho_{d}$, we can investigate the critical points of the Lyapunov function $V(\rho)=V\left(\rho, \rho_{d}\right)$ on $\mathcal{M}$.

Lemma III.1 (proof in [12]). For a given stationary target state $\rho_{d}$ the critical points of $V(\rho)=V\left(\rho, \rho_{d}\right)$ on $\mathcal{M}$ are such that $\left[\rho, \rho_{d}\right]=$ 0 .

If $\rho_{d}$ is generic, there are $n$ ! critical points of $V$ in total; if $\rho_{d}$ is non-generic, there are more critical points, forming different critical manifolds (see also [14]).

Example III.1. For a three-level system with $\rho_{d}=\operatorname{diag}\left(\frac{1}{4}, \frac{1}{4}, \frac{1}{2}\right)$ the set of $\rho \in \mathcal{M}-\left\{\rho_{d}\right\}$ that commute with $\rho_{d}$ has the form

$$
\rho_{0}=\left(\begin{array}{ccc}
w_{11} & w_{12} & 0 \\
w_{12}^{*} & w_{22} & 0 \\
0 & 0 & \frac{1}{4}
\end{array}\right),
$$

which forms a manifold $\mathcal{M}_{0}$ isomorphic to the Bloch sphere, on which $V$ assumes its global maximum, while $\rho=\rho_{d}$ corresponds to minimum of $V$.

Theorem III.1. Let $\rho_{d}$ be a given stationary target state. Then $\rho=$ $\rho_{d}$ is an isolated hyperbolic critical point of $V(\rho)$ corresponding to the global minimum and there are no other local or global minima.

Proof: $V(\rho)$ clearly assumes its global minimum for $\rho=\rho_{d}$. To show $\rho$ is a hyperbolic minimum of $V$ it suffices to show that it is a hyperbolic maximum of $J(\rho)=\operatorname{Tr}\left(\rho \rho_{d}\right)$. Choose a basis so that $\rho_{d}$ is diagonal and let $\rho=\rho_{0}$ be a critical point. Any point in a neighborhood of $\rho_{0}$ can be written as $\rho=e^{\vec{x} \cdot \vec{\sigma}} \rho_{0} e^{-\vec{x} \cdot \vec{\sigma}}$, where $\vec{\sigma}=\left\{\lambda_{k \ell}, \bar{\lambda}_{k \ell}, \lambda_{k}\right\}$ is the basis of the Lie algebra $\mathfrak{s u}(n)$ defined in the Appendix. Substituting this into $J$ gives $J=\operatorname{Tr}\left(e^{\vec{x} \cdot \vec{\sigma}} \rho_{0} e^{-\vec{x} \cdot \vec{\sigma}} \rho_{d}\right)$. To show that the critical point $\rho_{0}=\rho_{d}$ is a maximum of $J$, we need to find $n_{\mathcal{M}}=\operatorname{dim} \mathcal{M}$ independent directions in which $J$ is a local maximum. If we choose curves through $\rho_{0}$ with $\vec{x} \cdot \vec{\sigma}=\lambda_{k \ell} t$ then

$$
\begin{aligned}
J= & \operatorname{Tr}\left(\rho_{0} \rho_{d}\right)+t^{2}\left\{-\operatorname{Tr}\left(\rho_{0} \lambda_{k \ell}^{\dagger} \rho_{d} \lambda_{k \ell}\right)\right. \\
& \left.+\frac{1}{2} \operatorname{Tr}\left(\rho_{0} \rho_{d} \lambda_{k \ell}^{2}\right)+\frac{1}{2} \operatorname{Tr}\left(\rho_{0} \lambda_{k \ell}^{2} \rho_{d}\right)\right\}+\Theta\left(|t|^{3}\right) .
\end{aligned}
$$

The conjugate action of $\lambda_{k \ell}$ on $\rho=\rho_{d}$ on the $(k, \ell)$ subspace swaps the $k$-th and $\ell$-th diagonal elements. If we choose the curve with $\vec{x} \cdot \vec{\sigma}=\bar{\lambda}_{k \ell}$, we get a similar result. Hence the number of swaps that decrease the value of $J$ is

$$
2\left(n_{1} \sum_{\ell=2}^{k} n_{\ell}+\cdots+n_{k-1} n_{k}\right)=n^{2}-\sum_{\ell=1}^{k} n_{\ell}^{2}=\operatorname{dim} \mathcal{M} .
$$

Thus $\rho=\rho_{d}$ is a hyperbolic point of $J$, which is necessarily isolated. At other critical points, it is easy to see that there always exists some swap $\lambda_{k \ell}$ that increases the value of $J$, and hence all the other critical points cannot be local maxima of $J$, or local minima of $V$.

Remark III.1. For a given $\rho_{d}$, the critical points of $V(\rho)=V\left(\rho, \rho_{d}\right)$ form a finite number of isolated critical manifolds with $\rho_{d}$ as an isolated hyperbolic minimum. When $\rho_{d}$ becomes generic the critical manifolds are reduced to $n$ ! isolated hyperbolic critical points.

From Lemma III.1. we can see that for ideal Hamiltonian and stationary $\rho_{d}$, the LaSalle invariant set $E$ coincides with the critical points of $V(\rho)=V\left(\rho, \rho_{d}\right)$, and there are $p=\frac{n !}{n_{1} ! \cdots n_{k} !}$ stationary solutions including $\rho_{d}$, either isolated or located on an isolated manifold in $E$. In particular, since $\rho_{d}$ is isolated in $E$, the LaSalle invariance principle guarantees its local asymptotic stability, but if we want to investigate the asymptotic stability and convergence properties on a large scale, we must analyze the eigenvalues of the linearized dynamics at the other stationary states.

Theorem III.2. For ideal systems the stationary state $\rho_{d}$ is a hyperbolic sink of the dynamical system (2) and thus locally asymptotically stable, while all other stationary solutions have unstable manifolds. Hence, $\rho_{d}$ is almost globally asymptotically stable.

Proof: In order to analyze the stability at the stationary state we reformulate the dynamical system (2) in the Bloch representation

$$
\begin{aligned}
\dot{\vec{s}}(t) & =\left(A_{0}+f\left(\vec{s}, \vec{s}_{d}\right) A_{1}\right) \vec{s}(t) \\
\dot{\vec{s}}_{d}(t) & =A_{0} \vec{s}_{d}(t) \\
f\left(\vec{s}, \vec{s}_{d}\right) & ={\overrightarrow{s_{d}}}^{T} A_{1} \vec{s},
\end{aligned}
$$

where $\vec{s}=\left(s_{k}\right)_{k=1}^{n^{2}-1}$ with $s_{k}=\operatorname{Tr}\left(\sigma_{k} \rho\right)$, and $A_{0}$ and $A_{1}$ are the anti-symmetric matrices

$$
\begin{aligned}
& A_{0}\left(k, k^{\prime}\right)=\operatorname{Tr}\left(i H_{0}\left[\sigma_{k}, \sigma_{k^{\prime}}\right]\right), \\
& A_{1}\left(k, k^{\prime}\right)=\operatorname{Tr}\left(i H_{1}\left[\sigma_{k}, \sigma_{k^{\prime}}\right]\right),
\end{aligned}
$$

$\sigma_{k}$ being the elements of the basis of $\mathfrak{s u}(n)$ defined above. For stationary $\rho_{d}$, the dynamics is reduced to

$$
\begin{aligned}
\dot{\vec{s}}(t) & =\left(A_{0}+f(\vec{s}) A_{1}\right) \vec{s}(t) \\
f(\vec{s}) & ={\overrightarrow{s_{d}}}^{T} A_{1} \vec{s} .
\end{aligned}
$$

The linearized system near the critical point $\vec{s}_{0}$ is

$$
\dot{\vec{s}}=D_{f}\left(\vec{s}_{0}\right) \cdot\left(\vec{s}-\vec{s}_{0}\right),
$$

where $D_{f}\left(\vec{s}_{0}\right)=A_{0}+A_{1} \vec{s}_{0} \cdot{\overrightarrow{s_{d}}}^{T} A_{1}$ is a linear map defined on $\mathbb{R}^{n^{2}-1}$. (The map is defined on $\mathbb{R}^{n^{2}-1}$ as we neglect the $s_{0}$ coordinate of $\rho$, which is constant due to trace preservation.) In order to show $\vec{s}_{d}$ is hyperbolic, it suffices to show that there are $n_{\mathcal{M}}$ eigenvalues with nonzero real parts, corresponding to $n_{\mathcal{M}}$ eigenvectors in the tangent space of $\mathcal{M}$ at $\vec{s}_{d}$, denoted as $T_{\mathcal{M}}\left(\vec{s}_{d}\right)$. Let $S_{\mathcal{C}}$ and $S_{\mathcal{T}}$ be the subsets of $\mathbb{R}^{n^{2}-1}$ corresponding to the Cartan and non-Cartan subspaces $\mathcal{C}$ and $\mathcal{T}$ of $\mathfrak{s u}(n)=\mathcal{C} \oplus \mathcal{T}$. Since $\left[-i H_{0}, \rho_{d}\right]=0$ and $\left[-i H_{1}, \rho_{d}\right] \in i \mathcal{T}$, we have $A_{0} s_{d}=0$ and $A_{1} s_{d} \in S_{\mathcal{T}}$. Let $H_{1}=\left(b_{k \ell}\right)$, and $\vec{v}$ be a column vector consisting of $\frac{1}{2} n(n-1)$ blocks

$$
\vec{v}^{(k, \ell)}=\frac{\Delta_{k \ell}}{\sqrt{2}}\left(\begin{array}{c}
\Im\left(b_{k \ell}\right) \\
\Re\left(b_{k \ell}\right)
\end{array}\right) .
$$

Let $B=B_{0}-\vec{v} \vec{v}^{T}$ be the restriction of $D_{f}\left(\vec{s}_{d}\right)$ to the subspace $S_{\mathcal{T}}$ as before. Following a similar argument as in [12] it is easy to see that for $(k, \ell)$ such that $\Delta_{k \ell}=0$, the eigen-element $\left(\omega_{k \ell}, \vec{e}_{k \ell}\right)$ of $B_{0}$ is also an eigen-element of $B$ as $\vec{v}^{T} \vec{e}_{k \ell}=0$, and that $\vec{e}_{k \ell}$ corresponds to a direction orthogonal to the tangent space $T_{\mathcal{M}}\left(\vec{s}_{d}\right)$. The number 
of such $(k, \ell)$ is $\bar{N}=2 \sum_{\ell=1}^{k}\left(\begin{array}{c}n_{\ell} \\ 2\end{array}\right)$. We can therefore show that the remaining eigenvalues of $B$ with eigenvectors corresponding to the directions in $T_{\mathcal{M}}\left(\vec{s}_{d}\right)$ must have non-zero real parts. A simple counting argument shows that the number of these eigenvalues is $2\left(\begin{array}{l}n \\ 2\end{array}\right)-\bar{n}=\operatorname{dim}(\mathcal{M})$ and thus $\rho_{d}$ is a hyperbolic point. Since $\rho_{d}$ achieves the minimum of $V$, these eigenvalues must have negative real parts, i.e., $\rho_{d}$ must be a sink. Hence, there exists a neighborhood $\mathcal{N}$ of $\rho_{d}$ such that $\rho(0) \in \mathcal{N}$ will converge to $\rho_{d}$ for $t \rightarrow+\infty$, which establishes local asymptotic stability of $\rho_{d}$.

We can similarly show that any other stationary state $\rho_{0}$ that is isolated in $E$ is a hyperbolic fixed point, i.e., all eigenvalues of linearized system at $\rho_{0}$ have non-zero real parts. As $\rho_{0}$ is also an unstable critical point of the Lyapunov function $V$ by Theorem III.1. we can conclude there is an unstable manifold at $\rho_{0}$, as in the generic case [12]. When the stationary state $\rho_{0}$ lies on the critical manifold in $E$ then the linearized system at $\rho_{0}$ must again have an unstable manifold; for otherwise all eigenvalues of $D_{f}$ at $\rho_{0}$ would all have non-positive real parts, and $\rho_{0}$ would be a local minimum of $V$, contradicting Theorem III.1 Therefore, all stationary states except $\rho_{d}$ have an unstable manifold of positive dimension, and the dimensions of all stable manifolds at these unstable stationary states are less $\operatorname{dim} \mathcal{M}$. Hence, almost all non-stationary solutions will not converge to any of these unstable stationary states or their center manifolds, and therefore must converge to $\rho_{d}$. Thus, $\rho_{d}$ is almost globally asymptotically stable.

Example III.2. With $\rho_{d}=\operatorname{diag}\left(\frac{1}{4}, \frac{1}{4}, \frac{1}{2}\right)$ as in the previous example we have $n_{\mathcal{M}}=3^{2}-2^{2}-1=4$ and $E$ contains $\rho_{d}$ and two other stationary states $\rho_{1}=\operatorname{diag}\left(\frac{1}{4}, \frac{1}{2}, \frac{1}{4}\right)$ and $\rho_{2}=\operatorname{diag}\left(\frac{1}{2}, \frac{1}{4}, \frac{1}{4}\right)$. Analysis of the linearized dynamics shows that the two tangent vectors of the center manifold at $\rho_{\ell}$ are also the tangent vectors of E. Therefore, except for $\rho_{d}$, which is isolated, the points in $E$ form the center manifolds at the stationary states $\rho_{\ell}, \ell=1,2$.

For generic target states we showed in [12] that for non-ideal systems the target state itself generally becomes a center on an attractive center manifold, and the control becomes ineffective. For example, when $H_{0}$ is regular but not strongly regular then the LaSalle invariant set $E$ usually becomes much larger, forming a center manifold around the target state $\rho_{d}$, and most solutions $\rho(t)$ converge to points on this center manifold other than $\rho_{d}$. This is still true for most non-generic stationary $\rho_{d}$, for the same reasons, but unlike in the generic case, there are special target states that are asymptotically stable even if the Hamiltonian does not satisfy the criteria for ideal systems.

Example III.3. Consider the three-level system with $H_{1}$ fully connected but $H_{0}$ not strongly regular

$$
H_{0}=\left(\begin{array}{ccc}
-\omega & 0 & 0 \\
0 & 0 & 0 \\
0 & 0 & \omega
\end{array}\right), \quad H_{1}=\left(\begin{array}{ccc}
0 & 1 & 1 \\
1 & 0 & 1 \\
1 & 1 & 0
\end{array}\right) .
$$

Let $\rho_{d}=\operatorname{diag}\left(\alpha_{1}, \ldots, \alpha_{n}\right)$ be the stationary target state, $\rho=\left(\beta_{m n}\right)$ and $\Delta_{k \ell}=\alpha_{k}-\alpha_{\ell}$. Then

$$
\left[\rho, \rho_{d}\right]=\left(\begin{array}{ccc}
0 & -\beta_{12} \Delta_{12} & -\beta_{13} \Delta_{23} \\
\beta_{12}^{*} \Delta_{12} & 0 & -\beta_{23} \Delta_{23} \\
\beta_{13}^{*} \Delta_{23} & \beta_{23}^{*} \Delta_{23} & 0
\end{array}\right),
$$

but for the given Hamiltonians $H_{0}$ and $H_{1}$, we find that any point $\rho$ in the LaSalle invariant set satisfies

$$
\left[\rho, \rho_{d}\right]=\left(\begin{array}{ccc}
0 & \gamma & 0 \\
\gamma^{*} & 0 & -\gamma \\
0 & -\gamma^{*} & 0
\end{array}\right)
$$

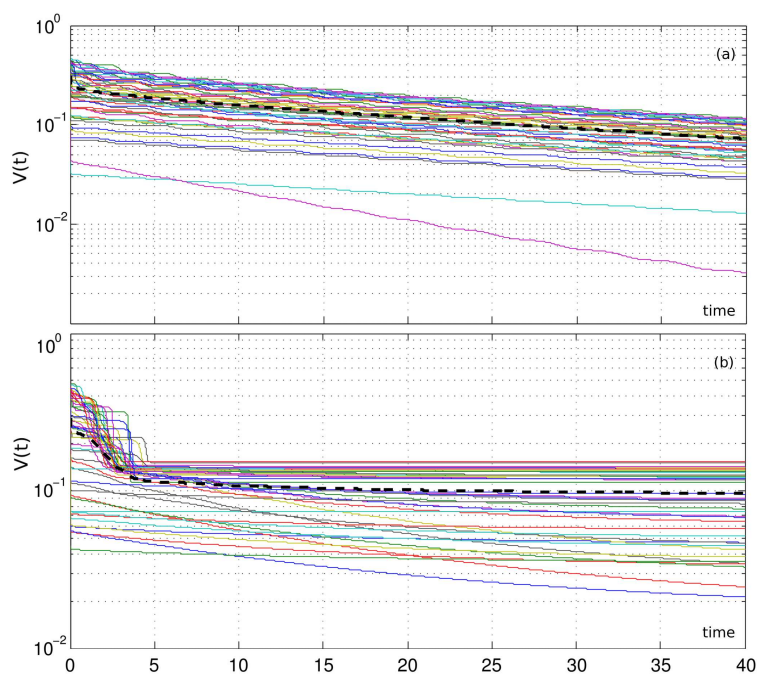

Fig. 1: Evolution of $V(t)=V\left(\rho(t), \rho_{d}(t)\right)$ with $y$-axis in logarithmic scale. Each graph shows $V(t)$ for $N=50$ different initial states $\rho(0)$ for a model system with $H_{1}$ fully connected and $H_{0}$ regular but not strongly regular. (a) For $\rho_{d}=\operatorname{diag}(1,0,0)$ all trajectories appear linear with negative slope in the semi-logarithmic plot, indicating exponential convergence; (b) for $\rho_{d}=\operatorname{diag}(0,1,0)$ most trajectories flat-line after some time at different non-zero distances, suggesting those trajectories do not converge to $\rho_{d}$ but its center manifold.

If $\rho_{d}=\operatorname{diag}(0,1,0)$, then any $\rho \in E$ must satisfy $\beta_{11}=\beta_{33}$, $\beta_{12}=\beta_{23},\left|\beta_{13}\right|=\beta_{11}$ and $\left|\beta_{12}\right|=\beta_{11}-2 \beta_{11}^{2}$. Analogous to the generic case, the points in LaSalle invariant set form a center manifold around $\rho_{d}$ and any solution starting outside $E$ will stop converging to $\rho_{d}$ after certain time, as shown in Fig. 1 (b).

However, if $\rho_{d}=\operatorname{diag}(1,0,0)$ then any $\rho \in E-\left\{\rho_{d}\right\}$ satisfies

$$
\rho=\left(\begin{array}{ccc}
0 & 0 & 0 \\
0 & \beta_{22} & \beta_{23} \\
0 & \beta_{23}^{*} & \beta_{33}
\end{array}\right)
$$

which corresponds to the maximum value of $V\left(\rho, \rho_{d}\right)$. Therefore, any solution starting outside $E$ satisfies $V\left(\rho(0), \rho_{d}\right)<V_{\max }$ and hence converges $\rho_{d}$, as shown in Fig. 1 a).

This example shows that the non-generic case differs from the generic one, but for most non-generic target states, a center manifold around the target state will still appear for non-ideal Hamiltonians, rendering the control design ineffective.

\section{Pseudo-Pure target States}

Pseudo-pure states, although a subset of measure zero of all possible states, deserve special consideration as they include the important special class of pure quantum states, and non-pure pseudopure states play an important role in applications such as ensemblebased quantum information processing. Moreover, for pseudo-pure target states the analysis simplifies, allowing us to derive the strong stability results for both stationary and non-stationary target states.

Any pseudo-pure state $\rho_{d}(0)$ can be written

$$
\rho_{d}(0)=w \Pi_{0}+\frac{1-w}{n-1} \Pi_{0}^{\perp}=w \Pi_{0}+u \Pi_{0}^{\perp}, \quad 0<w \leq 1,
$$

where $\Pi_{0}=\left|\Psi_{0}\right\rangle\left\langle\Psi_{0}\right|$ is a rank-1 projector onto some pure state $\left|\Psi_{0}\right\rangle$, and $\Pi_{0}^{\perp}$ is the projector onto the orthogonal subspace.

Theorem IV.1. Assuming ideal Hamiltonian, any solution $\rho(t)$ such that $V\left(\rho(0), \rho_{d}(0)\right)<V_{\max }$ converges to $\rho_{d}(t)$ as $t \rightarrow+\infty$ except 
when $\rho_{d}(0)=\left(r_{k \ell}\right)$ has a single pair of non-zero off-diagonal entries of the form $r_{k \ell}=\frac{1}{2}(w-u) e^{i \alpha}$ and $r_{k k}=r_{\ell \ell}=\frac{1}{2}(w+u)$. In the exceptional case any solution $\rho(t)$ converges to the orbit of $\rho_{d}(t)$ but in general $\rho(t) \nrightarrow \rho_{d}(t)$ as $t \rightarrow+\infty$ and $V\left(\rho, \rho_{d}\right)$ can take any limiting value between 0 and $V_{\max }$.

Proof: For any $\left(\rho_{1}, \rho_{2}\right) \in E, \rho_{1}$ and $\rho_{2}$ must also be pseudopure, with the same spectrum $\{w, u\}$, i.e., $\rho_{k}=w \Pi_{k}+u \Pi_{k}^{\perp}$ for $k=1,2$, and we have

$$
\left[\rho_{1}, \rho_{2}\right]=(w-u)^{2}\left[\Pi_{1}, \Pi_{2}\right] .
$$

Thus the LaSalle invariant set contains all points such that $M=$ $\left[\Pi_{1}, \Pi_{2}\right]$ is diagonal, according to Theorem $\Pi$ II.2 Let $\Pi_{k}=\left|\Psi_{k}\right\rangle\left\langle\Psi_{k}\right|$, $k=0,1,2$. Setting

$$
\begin{aligned}
& \left|\Psi_{1}\right\rangle=\left(a_{1} e^{i \alpha_{1}}, \ldots, a_{n} e^{i \alpha_{n}}\right)^{T}, \\
& \left|\Psi_{2}\right\rangle=\left(b_{1} e^{i \beta_{1}}, \ldots, b_{n} e^{i \beta_{n}}\right)^{T}
\end{aligned}
$$

we have $M=\left|\Psi_{1}\right\rangle\left\langle\Psi_{1} \mid \Psi_{2}\right\rangle\left\langle\Psi_{2}|-| \Psi_{2}\right\rangle\left\langle\Psi_{2} \mid \Psi_{1}\right\rangle\left\langle\Psi_{1}\right|$. For $\left(\rho_{1}, \rho_{2}\right) \in E$, the off-diagonal components of $M$ must vanish:

$$
M_{k \ell}=a_{k} b_{\ell} e^{i\left(\alpha_{k}-\beta_{\ell}\right)}\left\langle\Psi_{1} \mid \Psi_{2}\right\rangle-a_{\ell} b_{k} e^{i\left(\beta_{k}-\alpha_{\ell}\right)}\left\langle\Psi_{2} \mid \Psi_{1}\right\rangle=0,
$$

for all $k \neq \ell$. Let $\left\langle\Psi_{1} \mid \Psi_{2}\right\rangle=r e^{i \theta}$. We have the following two cases.

(a) $r=0$ i.e $\left\langle\Psi_{1} \mid \Psi_{2}\right\rangle=0$. In this case, $\left[\rho_{1}, \rho_{2}\right]=0$, and $V\left(\rho_{1}, \rho_{2}\right)=V_{\max }=(w-u)^{2}$.

(b) If $r \neq 0$ then (14) together with $M_{k k}=0$ gives:

$$
a_{k} b_{\ell}=a_{\ell} b_{k}, \quad \beta_{k}+\beta_{\ell}=\alpha_{k}+\alpha_{\ell}+2 \theta
$$

If $a_{k}=0$ then $0=a_{k} b_{\ell}=a_{\ell} b_{k}$ for $\ell \neq k$ and we must have $b_{k}=0$ as $a_{\ell}=0 \forall \ell$ is not allowed as $\vec{a}$ is a unit vector. Ditto for $b_{k}=0$. Let $I_{+}$be the set of all indices $k$ so that $a_{k}, b_{k} \neq 0$. Then

$$
\frac{a_{k}}{b_{k}}=\frac{a_{\ell}}{b_{\ell}}, \quad \forall k, \ell \in I_{+}
$$

and thus $\vec{a}=\gamma \vec{b}$, where $\vec{a}=\left(a_{k}\right)$ and $\vec{b}=\left(b_{k}\right)$. Since $\vec{a}$ and $\vec{b}$ are unit vectors in $\mathbb{R}_{+}^{n}, \gamma=1$ and $\vec{a}=\vec{b}$.

As for the phase equations (15), if $a_{k}=b_{k}=0$ then $M_{k \ell}=0$ is automatically satisfied, thus the only non-trivial equations are those for $k, \ell \in I_{+}$. If the set $I_{+}$contains $n_{1}>2$ indices then taking pairwise differences of the $n_{1}\left(n_{1}-1\right) / 2$ non-trivial phase equations and fixing the global phase of $\left|\Psi_{k}\right\rangle$ by setting $\alpha_{n_{1}}=\beta_{n_{1}}=0$ shows that $\vec{\alpha}=\vec{\beta}$. For example, suppose $I_{+}=\{1,2,3\}$ then we have 3 non-trivial phase equations

$$
\begin{aligned}
& \beta_{1}+\beta_{2}=\alpha_{1}+\alpha_{2}+2 \theta, \\
& \beta_{1}+\beta_{3}=\alpha_{1}+\alpha_{3}+2 \theta, \\
& \beta_{2}+\beta_{3}=\alpha_{2}+\alpha_{3}+2 \theta,
\end{aligned}
$$

taking pairwise differences gives

$$
\begin{aligned}
& \beta_{2}-\beta_{3}=\alpha_{2}-\alpha_{3}, \\
& \beta_{1}-\beta_{3}=\alpha_{1}-\alpha_{3}, \\
& \beta_{1}-\beta_{2}=\alpha_{1}-\alpha_{2},
\end{aligned}
$$

and setting $\alpha_{3}=\beta_{3}=0$ shows that we must have $\alpha_{2}=\beta_{2}$ and $\alpha_{3}=\beta_{3}$. Thus, together with $\vec{a}=\vec{b}$ we have $\rho_{1}=\rho_{2}$. If $I_{+}$ contains only a single element then $\left|\Psi_{1}\right\rangle$ and $\left|\Psi_{2}\right\rangle$ differ at most by a global phase and again $\rho_{1}=\rho_{2}$ follows. Incidentally, note that for $\left|\Psi_{1}\right\rangle=\left|\Psi_{2}\right\rangle$ we have $\left\langle\Psi_{1} \mid \Psi_{2}\right\rangle=1$, i.e., $r=1, \theta=0$.

The only exceptional case arises when $I_{+}$contains exactly two elements, say $\{1,2\}$, as in this case there is only a single phase equation $\beta_{1}+\beta_{2}=\alpha_{1}+\alpha_{2}+2 \theta$, and thus even fixing the global phase by setting $\alpha_{2}=\beta_{2}=0$, only yields $\beta_{1}-\alpha_{1}=2 \theta$. This combined with $\vec{a}=\vec{b}$ gives

$$
r e^{i \theta}=\left\langle\Psi_{1} \mid \Psi_{2}\right\rangle=a_{1}^{2} e^{2 i \theta}+a_{2}^{2}
$$

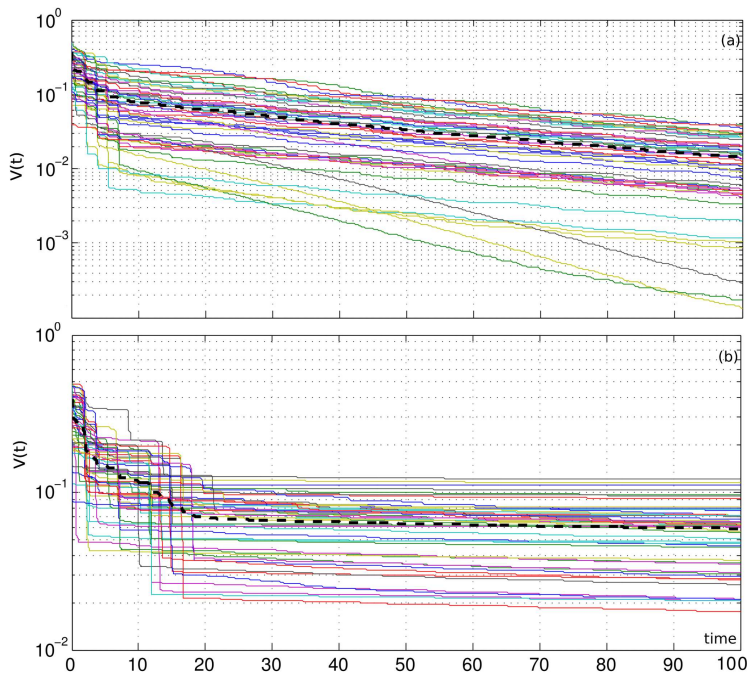

Fig. 2: $V(t)=V\left(\rho(t), \rho_{d}(t)\right)$ with $y$-axis in logarithmic scale. Each graph shows $V(t)$ for $N=50$ different initial states $\rho(0)$ for a twoqubit system with ideal Hamiltonian. (a) For the target state $|\Psi\rangle=$ $\frac{1}{\sqrt{5}}(1,0,0,2)^{T}$ all trajectories converge exponentially to the target. (b) For the target state $|\Psi\rangle=\frac{1}{\sqrt{2}}(1,0,0,1)^{T}$, most trajectories stop converging after some finite time and $\lim _{t \rightarrow \infty} V(t)>0$.

and thus $a_{1}^{2} e^{i \theta}+a_{2}^{2} e^{-i \theta}=r$ or $2 i \sin \theta\left(a_{1}^{2}-a_{2}^{2}\right)=0$. Therefore, either $\theta=0$ or $a_{1}=a_{2}$. If $\theta=0$ then $\vec{\alpha}=\vec{\beta}$ and $\rho_{1}=\rho_{2}$, which is one possible solution in $E$. If $\theta \neq 0$, then any $\left(\rho_{1}, \rho_{2}\right)$ satisfying

$$
\begin{aligned}
& \left|\Psi_{1}\right\rangle=2^{-1 / 2}\left(1, e^{i \alpha}, 0, \ldots, 0\right)^{T} \\
& \left|\Psi_{2}\right\rangle=2^{-1 / 2}\left(1, e^{i \beta}, 0, \ldots, 0\right)^{T}
\end{aligned}
$$

with $\beta-\alpha=2 \theta$ is also in $E$. Hence, if $\left|\Psi_{0}\right\rangle$ has only two nonzero components with equal norm, e.g., if

$$
\rho_{d}(0)=\left(\begin{array}{ccccc}
r_{11} & r_{12} & 0 & \ldots & 0 \\
r_{12}^{\dagger} & r_{11} & 0 & \ldots & 0 \\
0 & 0 & u & & \\
\vdots & \vdots & & \ddots & \\
0 & 0 & & & u
\end{array}\right)
$$

with $r_{11}=\frac{1}{2}(w+u), r_{12}=\frac{1}{2}(w-u) e^{i \alpha}$, and $\left|\Psi_{0}\right\rangle=$ $2^{1 / 2}\left(1, e^{i \alpha}, 0, \ldots, 0\right)^{T}$, then $E$ contains all points $\left(\rho_{1}, \rho_{2}\right)$ satisfying (17), which includes $\rho_{1}=\rho_{2}$ and $\rho_{1} \perp \rho_{2}$. Since $\rho_{1}$ and $\rho_{2}$ lie on the orbit of $\rho_{d}(0)$, any solution $\rho(t)$ will converge to this orbit but we cannot guarantee $\rho(t) \rightarrow \rho_{d}(t)$ as $t \rightarrow+\infty$. For all other $\rho_{d}(0) E$ contains only points with either $\rho_{1}=\rho_{2}$ or $\rho_{1} \perp \rho_{2}$, corresponding to $V=0$ and $V=V_{\max }$, respectively, and since $V$ is non-increasing, any solution $\rho(t)$ with $V\left(\rho(0), \rho_{d}(0)\right)<V_{\max }$ will converge to $\rho_{d}(t)$ as $t \rightarrow+\infty$.

\section{APPLICATIONS FOR TWO-QUBIT SYSTEMS}

Four-level (or two-qubit) systems are interesting for many reasons, but for our purposes four-level systems are of interest as $n=4$ is the lowest dimension that admits states that are neither generic nor pseudo-pure. Any stationary state $\rho_{d}$ has one of the following forms:

- generic: $\rho_{d}=\operatorname{diag}(a, b, c, d)$

- pseudo-pure: $\rho_{d}=\operatorname{diag}(a, b, b, b)$

- other: $\rho_{d}=\operatorname{diag}(a, a, b, b)$

where $a, b, c, d$ are distinct. The first two cases correspond to the cases discussed above and in [12]. In the third case, $\rho_{d}=\operatorname{diag}(a, a, b, b)$, 
the state manifold $\mathcal{M}$ is homeomorphic to the flag manifold $\mathbb{U}(4) / \mathbb{U}(2) \times \mathbb{U}(2)$, or $\mathbb{S} U(4) /\left\{\exp (\sigma): \sigma \in \mathcal{C} \oplus \mathcal{T}_{12} \oplus \mathcal{T}_{34}\right\}$ and has real dimension $n_{\mathcal{M}}=8$. Let $(a, a, b, b)$ represent $\operatorname{diag}(a, a, b, b)$, etc. There are six stationary solutions: $(a, a, b, b),(a, b, a, b),(a, b, b, a)$, $(b, a, a, b),(b, a, b, a)$ and $(b, b, a, a)$. According to the results in Section III the critical points of $V(\rho)=V\left(\rho, \rho_{d}\right)$ consist of six components: two isolated hyperbolic critical points, $(a, a, b, b)$ and $(b, b, a, a)$, corresponding to the global minimum $V=0$ and maximum $V=2(a-b)^{2}$, respectively, and four critical manifolds homeomorphic to $S^{2} \times S^{2}$ on which $V$ assumes the intermediate value $(a-b)^{2}$.

Calculating the $D_{f}\left(\vec{s}_{0}\right)$ restricted to $S_{\mathcal{T}}$ from the linearized dynamics (9) at each stationary state $\rho_{0}$, as in the proof of Theorem III.2 shows that for ideal systems, the dimension of the stable manifold at $\rho_{d}=\operatorname{diag}(a, a, b, b)$ is $n_{s}=8=n_{\mathcal{M}}$, while at $(b, b, a, a)$, the dimension the unstable manifold $n_{u}=8=n_{\mathcal{M}}$. Thus these two points are a hyperbolic sink and source, respectively, with $V_{\min }=0$ and $V_{\max }=2(a-b)^{2}$. At any of the other four stationary states, i.e., $(a, b, a, b),(a, b, b, a),(b, a, b, a)$ and $(b, b, a, a)$, we have $V_{0}=(a-b)^{2}$, a stable and unstable manifold of dimension 2 and two pairs of purely imaginary eigenvalues, showing that these intermediate critical points are centers, but the dimension of the corresponding center manifolds is less than $n_{\mathcal{M}}$, and each of these centers has an unstable manifold. Therefore, the center manifolds and the set of states that are attracted to them, form a set of measure zero in the state space, and even in the vicinity of a center manifold, many states will converge to $\rho_{d}$. Thus we can say that $\rho_{d}$ is almost globally asymptotically stable.

While this suggests that the Lyapunov design is mostly effective, at least for ideal Hamiltonian, unfortunately, it still fails for some of the most interesting problems. As an example, consider the problem of Bell state generation, i.e., tracking the trajectory of a maximally entangled state such as $\left|\Psi_{+}\right\rangle=\frac{1}{\sqrt{2}}(|00\rangle+|11\rangle)$, which is important in quantum information. Setting $\rho_{d}(0)=\left|\Psi_{+}\right\rangle\left\langle\Psi_{+}\right|$ we see immediately that we cannot track this state because $\rho_{d}(0)$ satisfies the conditions of Theorem IV.1 for which the method fails: Since $\rho_{d}(0)$ is pure we have $w=1$ and $u=0$ and taking the standard Pauli product basis we have $\rho_{d}(0)=\left(r_{k \ell}\right)$ with $r_{11}=r_{14}=r_{41}=r_{44}=\frac{1}{2}$ and $r_{k \ell}=0$ otherwise. Fig. 2(b) shows that most initial states converge to points in the invariant set $E$ that are finite distance from $\rho_{d}$. We could console ourselves that the method does work well for most other target states such as the example in Fig. 2 a). However, the effectiveness of the method in these cases is predicated on the assumption of ideal Hamiltonians, which excludes most typical physical Hamiltonians such as $H_{0}=0.1 \sigma_{z} \otimes \sigma_{z}$ and $H_{1}=\sigma_{x} \otimes \mathbb{I}+0.9 \mathbb{I} \otimes \sigma_{x}$ for a system with constant Ising coupling and local $x$-rotations. In this case $H_{0}$ is not even regular and many of the off-diagonal elements of $H_{1}$ are zero, and the invariant set $E$ becomes huge, rendering the method ineffective.

\section{CONCLUSION}

We have extended our previous work on Lyapunov control for generic quantum states to the non-generic case. Although the LaSalle invariant set is now much larger, containing critical manifolds, we find that for ideal Hamiltonian, the stationary target state is still almost globally asymptotically stable. For non-ideal systems we find that in general the target state changes from an isolated asymptotically stable minimum to a center on a center manifold as in the generic case, but unlike in the generic case, there are special cases where a non-generic target state remains asymptotically stable under non-ideal Hamiltonian, though these states are exceptional. For the important class of pseudo-pure states, necessary and sufficient conditions for convergence to the target state for both stationary and non-stationary cases are derived by alternative mean. Finally, application to twoqubit systems shows that even for ideal systems the small set of (non-stationary) states that are non-trackable using Lyapunov control includes some of the most interesting cases such as CAT states and maximally-entangled Bell states.

\section{ACKNOWLEDGMENTS}

XW is supported by the Cambridge Overseas Trust. SGS is acknowledges funding from an EPSRC Advanced Research Fellowship, Hitachi, EU Knowledge Transfer Programme MTDK-CT-2004509223 and NSF Grant PHY05-51164.

\section{APPENDIX}

A standard basis for the Lie algebra $\mathfrak{s u}(n)$ is given by $\left\{\lambda_{k \ell}, \bar{\lambda}_{k \ell}, \lambda_{k}\right\}$ for $1 \leq k<\ell \leq n$, where

$$
\begin{aligned}
& \lambda_{k}=i\left(\hat{e}_{k k}-\hat{e}_{k+1, k+1}\right) \\
& \lambda_{k \ell}=i\left(\hat{e}_{k \ell}+\hat{e}_{\ell k}\right), \quad \bar{\lambda}_{k \ell}=\left(\hat{e}_{k \ell}-\hat{e}_{\ell k}\right)
\end{aligned}
$$

and the $(k, \ell)^{\text {th }}$ entry of the matrix $\hat{e}_{m n}$ is $\delta_{k m} \delta_{\ell n}$, and $i=\sqrt{-1}$. We have the useful identities

$$
\begin{aligned}
& \operatorname{Tr}\left(\lambda_{k \ell} \lambda_{k^{\prime} \ell^{\prime}}\right)=\operatorname{Tr}\left(\bar{\lambda}_{k \ell} \bar{\lambda}_{k^{\prime} \ell^{\prime}}\right)=-2 \delta_{k k^{\prime}} \delta_{\ell \ell^{\prime}} \\
& \operatorname{Tr}\left(\lambda_{k \ell} \bar{\lambda}_{k^{\prime} \ell^{\prime}}\right)=0
\end{aligned}
$$

and for a diagonal matrix $D=\sum_{k=1}^{n} d_{k} \hat{e}_{k k}$ we have $\left[D, \lambda_{k}\right]=0$,

$$
\left[D, \lambda_{k \ell}\right]=+i\left(d_{k}-d_{\ell}\right) \bar{\lambda}_{k \ell}, \quad\left[D, \bar{\lambda}_{k \ell}\right]=-i\left(d_{k}-d_{\ell}\right) \lambda_{k \ell} .
$$

The basis (19) is not orthonormal but we can define an equivalent orthonormal basis by normalizing the $n^{2}-n$ non-Cartan generators $\frac{1}{\sqrt{2}} \lambda_{k \ell}$ and $\frac{1}{\sqrt{2}} \bar{\lambda}_{k \ell}$, and defining the $n-1$ orthonormal generators for the Cartan subalgebra $\sigma_{n^{2}-n+r}=i[r(r+$ $1)]^{-1 / 2}\left(\sum_{s=1}^{r} \hat{e}_{s s}-r \hat{e}_{r+1, r+1}\right)$ for $r=1, \ldots, n-1$.

\section{REFERENCES}

[1] P. Vettori, "On the convergence of a feedback control strategy for multilevel quantum systems," in Proc. MTNS 2002, 21350.

[2] A. Ferrante, M. Pavon, and G. Raccanelli, "Driving the propagator of a spin system: a feedback approach," in Proc. 41st IEEE CDC 2002, 46-50.

[3] S. Grivopoulos and B. Bamieh, "Lyapunov-based control of quantum systems," In Proc. 42nd IEEE CDC 2003, 431-438.

[4] M. Mirrahimi and P. Rouchon, "Trajectory generation for quantum systems based on Lyapunov techniques," In Proc. NOLCOS 2004, 291.

[5] M. Mirrahimi and P. Rouchon, "Trajectory tracking for quantum systems: A Lyapunov approach," In Proc. MTNS 2004.

[6] M. Mirrahimi, P. Rouchon and G. Turinici, "Lyapunov control of bilinear Schrodinger equations," Automatica, vol. 41, pp. 1987-1994, 2005.

[7] K. Beauchard, J.-M. Coron, M. Mirrahimi and P. Rouchon, "Implicit Lyapunov control of finite dimensional Schrodinger equations," System Control Lett., vol. 56, pp. 388-395, 2007.

[8] M. Mirrahimi and R. Van Handel, "Stabilizing feedback controls for quantum systems," SIAM J. Control Optim., vol. 46, pp. 445-467, 2007.

[9] C. Altafini, "Feedback control of spin systems," Quantum Information Processing, vol. 6, pp. 9-36, 2007

[10] C. Altafini, "Feedback stabilization of quantum ensembles: a global convergence analysis on complex flag manifolds," IEEE Trans. Autom. Control, vol. 52, pp. 2019-2031, 2007.

[11] D. D'Alessandro, "Introduction to Quantum Control and Dynamics," CRC Press, 2007.

[12] X. Wang and S. G. Schirmer, "Analysis of Lyapunov Method for Control of Quantum States," IEEE TAC submitted, 2008.

[13] J. LaSalle and S. Lefschetz, "Stability by Liapunov's Direct Method with Applications," New York: Academic Press, 1961.

[14] R. Wu, H. Rabitz and M. Hsieh, "Characterization of the critical submanifolds in the quantum ensemble control landscapes," J. Phys. A, vol. 41, 015006, 2008. 